\title{
El Aprendizaje Basado en Problemas (ABP) en el desarrollo de la inteligencia emocional de estudiantes universitarios
}

\section{Problem Based Learning (PBL) in the Development of Emotional Intelligence of University Students}

\author{
Carlos Luy-Montejo \\ Universidad Privada del Norte, Lima, Perú. \\ ORCID: https://orcid.org/0000-0003-0824-7959
}

Recibido 19-09-18 Revisado 25-11-18 Aprobado 28-01-19 En línea 12-02-19

Correspondencia

Email: fracarlitos@gmail.com

\section{Citar como:}




\section{Resumen}

El presente artículo trata sobre el efecto del Aprendizaje Basado en Problemas (ABP) en el desarrollo de la inteligencia emocional de estudiantes del primer ciclo en una universidad privada de Lima. Para lograr este propósito se realizó una investigación de tipo aplicado, empleando el método experimental (diseño cuasi-experimental), dado que se manipulará la variable independiente $(\mathrm{ABP})$ para que produzca un efecto sobre la variable dependiente (Inteligencia Emocional), la cuál será medida. Para ello, se tomó una muestra de 48 estudiantes distribuidos en dos grupos: uno de control (24) y otro experimental (24) de una población total de 1281 ingresantes. Para la recolección de datos, se empleó el test de Inventario de Inteligencia Emocional de Bar-On (I-CE) como prueba de entrada (pretest), aplicándose la metodología del ABP durante 16 sesiones de aprendizaje al grupo experimental, mientras que para el grupo de control se utilizaron metodologías de aprendizaje tradicionales; para luego efectuarse las mediciones con el mismo test de BarOn como prueba de salida (postest) para ambos grupos. Los resultados mostraron que hubo influencia significativa del ABP en el desarrollo de la inteligencia emocional de los estudiantes objeto del estudio.

Palabras clave: Aprendizaje basado en problemas; Metodología activa de aprendizaje; Competencias emocionales; Inteligencia emocional; Habilidades blandas.

\section{Summary}

This article deals with the effect of Problem Based Learning (PBL) in the development of the emotional intelligence of first-semester students in a private university in Lima. To achieve this purpose, an applied research was carried out, using the experimental method (quasi-experimental design), given that the independent variable (PBL) will be manipulated so that it produces an effect on the dependent variable (Emotional Intelligence), which will be measured. To do this, a sample of 48 students was taken, it was divided into two groups: one of control (24) and the other one experimental (24) of a total population of 1281 freshmen. For the collection of data, the BarOn Emotional Intelligence Inventory (I-CE) test was used as a pretest. PBL methodology was then applied throughout 16 learning sessions to the experimental group, while the control group received traditional learning methodologies. The same Bar-on test was then used as posttest to conduct measures for both groups. The results showed that there was significant influence of the PBL in the development of the emotional intelligence of the students of study.

Keywords: Problem-Based Learning; Active Learning Methodology; Emotional Competencies; Emotional Intelligence; Soft Skills.

\section{Introducción}

Las exigencias del contexto nacional e internacional de formar recursos humanos que se encuentren preparados para enfrentar las nuevas necesidades del mercado laboral y el contexto globalizado y multicultural de nuestras sociedades, implican una educación integral, no solamente como la encargada de impartir instrucción o transmitir conocimiento, sino una educación que favorezca el desarrollo de todas las capacidades para la realización del proyecto personal de vida y como un medio para transformar la realidad.

Es por ello, que las demandas del mercado laboral requieren una educación superior capaz de formar a estudiantes con un potencial desempeño a nivel humano y profesional. Se buscan 
nuevas maneras de preparar a los estudiantes de educación superior, para lograr una exitosa inserción laboral, no solo preparándolos a nivel cognitivo, sino en el desarrollo de habilidades blandas (soft skills) que son sumamente necesarias en el perfil actual de los profesionales. De hecho, se han puesto en marcha en los últimos años amplios proyectos de reforma educativa que buscan nuevas formas de atender dichas demandas y exigencias de la sociedad actual. De esa forma, con el fortalecimiento de competencias o habilidades ligadas a la inteligencia emocional, se podrá optimizar el perfil del estudiante de educación superior y como consecuencia de ello podrá convertirse en un individuo íntegro capaz de poder adaptarse e interrelacionarse positivamente con el medio, dinámico y cambiante.

Sin embargo, frente a la demanda de profesionales con competencias a nivel personal, social y académico, nos enfrentamos a estudiantes que a nivel educación superior siguen "arrastrando" esos vacíos que la educación básica regular no logra suplir: estudiantes con serias dificultades a nivel personal (baja autoestima, poca autorregulación y motivación), con tensiones frecuentes en sus relaciones y contactos personales (escaso desarrollo de sus habilidades sociales y empatía), poco o nada implicados en las actividades y participación en clase (metodologías centradas en la enseñanza, memorísticas y solo preocupadas del desarrollo a nivel cognitivo); todo esto ha conllevado a que la deserción académica sea una constante a nivel de instituciones de educación superior, así como el alto grado de repitencia.

Por lo anterior, se observa la necesidad de llevar a cabo una investigación sobre el efecto de metodologías activas de aprendizaje que comprometan al docente en un rol proactivo en aula, como verdadero acompañante del proceso de enseñanza-aprendizaje, que promueva el aprendizaje autónomo y el desarrollo de la inteligencia emocional de los estudiantes, como fundamento vital de todo desarrollo integral de nuestros futuros profesionales. Se propone un programa de intervención a nivel pedagógico, de manera presencial en aula y de aprendizaje cooperativo, el Aprendizaje Basado en Problemas (ABP), para lograr, como objetivo de la investigación, un efecto significativo en el desarrollo de los componentes de la Inteligencia Emocional, pero sobretodo, para asegurar una formación integral de los estudiantes que aseguren el éxito a nivel personal, académico y profesional. La pregunta a la que se tuvo que dar respuesta fue: ¿Cuál es el efecto del Aprendizaje Basado en Problemas en la inteligencia emocional en un grupo de estudiantes del primer ciclo de una universidad privada de Lima? La hipótesis sustantiva de la presente investigación parte del supuesto que una intervención pedagógica basada en la metodología del ABP desarrollaría más significativamente los componentes de la Inteligencia Emocional, en comparación a la metodología de aprendizaje tradicional.

\section{Aprendizaje Basado en Problemas.}

El aprendizaje basado en problemas (ABP) es "un tipo de metodología activa, de enseñanza, centrada en el estudiante, que se caracteriza por producir el aprendizaje del estudiante en el contexto de la solución de un problema auténtico" (Marra, Jonassen, Palmer \& Luft, 2014, p.221).

El Aprendizaje Basado en Problemas se fundamentó en dos argumentos conceptuales y teóricos. Uno de ellos es la obra del filósofo de la educación John Dewey, quien subrayó la importancia de aprender mediante la experiencia. Según Dewey, en esa experiencia del mundo real, los estudiantes encuentran un problema que estimula su pensamiento, se informan para plantear soluciones tentativas al problema y la aplicación los ayuda a comprobar su conocimiento. Por otro lado, el ABP recoge la teoría sociocultural de Vigotsky, quien subrayó la importancia de la participación del estudiante en comunidades de aprendizaje cognitivo, donde el estudiante 
intercambia y compara ideas con la de los otros, interactuando activamente para resolver problemas y el profesor dirige sus esfuerzos (Eggen \& Kauchak, 2015).

Marra, Jonassen, Palmer y Luft, (2014) propusieron que las características principales de un entorno de aprendizaje basado en problemas son las siguientes: (1) el aprendizaje centrado en el problema (contenidos y habilidades a ser aprendidas organizadas alrededor de problemas reales auténticos), (2) el aprendizaje centrado en el estudiante (se despliegan una serie de procesos cognitivos y afectivos para investigar y resolver el problema), (3) la auto-dirección (se demanda a los estudiantes asumir la responsabilidad de: identificar los objetivos de aprendizaje, planificar el recojo de información y realizar la búsqueda, procesamiento e integración de la información), (4) la auto-reflexión (se propicia que los estudiantes monitoreen su comprensión y aprendizaje para ajustar sus estrategias), (5) el trabajo colaborativo (se estimula el intercambio, diálogo y discusión entre pares) y (6) el andamiaje del docente (se actúa como facilitador cuyo rol fundamental es modelar y guiar procesos de razonamiento, de búsqueda e integración de información, facilitar procesos grupales y formular preguntas para indagar sobre la exactitud, pertinencia y profundidad de análisis de la información).

Estas características del ABP se ajustan de manera óptima al aprendizaje en estudiantes universitarios, ya que "cuando el alumno accede al sistema universitario se enfrenta a un entramado complejo de demandas para las cuales no está siempre preparado, como puede ser la autonomía en el proceso de aprendizaje, el desarrollo de habilidades sociales, etc" (Lino, 2015, p. 28). Es así que se consideró de suma importancia el uso de metodologías activas de aprendizaje, especialmente el ABP, para lograr la orientación que se le brinda al estudiante, ya que, según el punto de vista de este autor, las dificultades del alumnado universitario son personales, familiares o económicas, lo que deriva en inseguridad y temor.

Lino (2015) planteó que se debe poner énfasis en el desarrollo de las habilidades sociales, estrategias de afrontamiento y la búsqueda de apoyo social que contrarrestan el posible abandono universitario y/o la insatisfacción en esta etapa, sobretodo en estudiantes ingresantes. En este sentido, el ABP fomenta tanto la inteligencia emocional interpersonal, como las habilidades sociales, que incluye ser capaces de expresar adecuadamente nuestras emociones, comprenderlas, aceptarlas y reducir las negativas, como la ira. Por lo tanto, el desarrollo del Inteligencia Emocional, fomentada por el ABP, "parece aportar cierto grado explicativo en el ajuste socioacadémico del estudiante, especialmente en lo que se refiere a rendimiento académico y conductas disruptivas en el aula, así como respecto a otras variables como el estrés y la ansiedad de los alumnos, el consumo de tabaco y alcohol, el optimismo o la madurez vocacional" (Pena \& Repetto, 2008, citados por Gutiérrez \& Expósito, 2015, p. 45)

\section{Fases para la implementación del Aprendizaje Basado en Problemas.}

Diaz Barriga y Hernández (2010) propones tres fases para la implementación de esta propuesta pedagógica: 
Tabla 1.

Fases de la propuesta pedagógica del ABP

\section{Fases}

a) Preparación de la situación del ABP.

b) Establecimiento de la situación del ABP entre los estudiantes.

c) Proceso de resolución de problemas.
Sub-fases

- Identificar los hechos e ideas relevantes que se convertirán en la situación problemática.

- Definir de manera clara los propósitos del ABP.

- Elaborar los sílabos y el instrumento de evaluación.

- Presentación y explicación de la situación problemática y los instrumentos de evaluación a los estudiantes.

- Formar los grupos de trabajo.

- Identificar los primeros intentos de solución del problema por parte de los estudiantes.

- Identificar y analizar: conocimientos previos, aquello que necesitan aprender y aquellos que aprenderán.

- Planteamiento de objetivos.

- Actividades colaborativas para la búsqueda de información que permitan plantear la estrategia de solución.

- Planteamiento de la planificación e implantación de la estrategia de solución.

- Comunicación de resultados al grupo de clase y docente.

\section{Pasos para la aplicación del Aprendizaje Basado en Problemas.}

Según Eggen y Kauchak (2015) la aplicación del ABP en aula debe ser desarrollada en 5 etapas:

Etapa 1: Identificar una pregunta

Una investigación empieza cuando se identifica una pregunta hecha para llamar la atención y construir un reto para los estudiantes. Se busca en esta primera etapa suscitar la curiosidad en el estudiante, aprovechándose los efectos motivadores de indagación y desafío.

\section{Etapa 2: Generar hipótesis}

Una vez establecida la pregunta, el grupo está listo para intentar darle respuesta. Una hipótesis es una respuesta tentativa a una pregunta o solución a un problema que puede verificarse con información. La generación de la hipótesis activa el conocimiento anterior e inicia el proceso de producción de esquemas.

\section{Etapa 3: Acopio de información}

Las hipótesis guían el proceso de acopio de información. Éste promueve la metacognición cuando los alumnos planean las estrategias para reunir información. Dentro de esta fase es importante que el estudiante desarrolle habilidades para organizar y presentar la información a través de cuadros, esquemas o gráficos. 


\section{Etapa 4: Evaluación de hipótesis}

En esta fase, los estudiantes son responsables de evaluar sus hipótesis con base en los datos. El hecho de encontrar, por ejemplo, datos contradictorios es, en si mismo, una experiencia importante para los estudiantes. En esta vida pocas cosas son claras e inconfundibles, y cuanta más experiencia tengan los estudiantes en enfrentarse a su ambigüedad, que requiere conclusiones tentativas y no dogmáticas, mejor preparados saldrán al mundo.

\section{Etapa 5: Generalizar}

El cierre del contenido en una clase de aprendizaje basado en problemas se realiza cuando los estudiantes generalizan tentativamente acerca de los resultados con base en la información. Con el tiempo, ellos desarrollan una tolerancia a la complejidad y a la ambigüedad que puede ayudarles a comprender y a hacer frente a las complejidades de la vida. Finalmente, cabe indicar que, al término de la última etapa, el Aprendizaje Basado en Problemas ofrece también oportunidades para que el estudiante reflexione sobre el proceso, y así puede esperarse que éste sea capaz de transferirlo a otras actividades del aula y también a su vida cotidiana, lo cual es su propia forma de generalizar.

\section{Ventajas del método ABP para el desarrollo de competencias en estudiantes universitarios.}

Escribano y Del Valle (2010) señalaron el reconocimiento de las ventajas del ABP sobre los métodos convencionales de aprendizaje que han promovido su difusión y aplicación en diversas disciplinas a nivel educación superior. Entre las ventajas señalas por estos autores se pueden enfatizar:

- La motivación como "voluntad de aprender" como lo señaló Bruner, es estimulada por el $\mathrm{ABP}$, ya que invita al estudiante a involucrarse más en el aprendizaje debido a que siente la posibilidad de interactuar con la realidad y a observar los resultados de dicha interacción.

- El estudiante, mediante esta metodología, logra establecer conexión sustantiva entre la información que va recibiendo y el conocimiento previo que posee, produciéndose un aprendizaje más significativo; este modo de aprender refuerza incluso su interés por seguir investigando también fuera de aula.

- La integración del conocimiento posibilita mayor retención y transferencia del conocimiento. La metodología del ABP permite desde la práctica, la detección de errores o inconsistencias teóricas lo que se perfila como una de las estrategias más propicias para la construcción de un aprendizaje que establece contacto con las concepciones previas del estudiante y que contribuye a transformarlas.

- El aprendizaje que se apoya en esta metodología estimula el pensamiento crítico y creativo, es decir, estimula la adquisición de habilidades para identificar problemas y ofrecer soluciones adecuadas a los mismos.

- El estudiante, mediante la metodología del ABP, logra lo que se conoce como la integración del conocimiento. El conocimiento de las diferentes disciplinas se integra para dar solución al problema sobre el cual se está trabajando, de tal modo, que el aprendizaje no se da en fracciones, sino de manera integral y dinámica.

- El método de ABP promueve la interacción incrementando habilidades interpersonales como: el trabajo en equipo, la evaluación de los compañeros, la presentación y defensa 
de los trabajos. Esta metodología interactiva permite desarrollar, extender y profundizar las habilidades interpersonales: los estudiantes tienen la oportunidad de compartir sus descubrimientos, y se brindan apoyos para resolver los problemas y trabajar en proyectos conjuntos.

- Se promueve la evaluación formativa, en un sistema de autoevaluación de los estudiantes y la coevaluación, que permite identificar y corregir errores, así como asegurar el alcance de metas personales y comunes, mediante la retroinformación constructiva.

Finalmente, con respecto a la metodología de Aprendizaje Basado en Problemas ABP que será empleada en el marco del programa de intervención, se puede señalar que esta propuesta metodológica tiene como eje central que el estudiante sea un ente activo de su propio aprendizaje y el docente una guía que promueva estos aprendizajes. Existen diversas propuestas pedagógicas de metodologías activas de aprendizaje centradas en el estudiante, las cuales tienen la intención de promover aprendizajes situados, experienciales y auténticos en los alumnos, que les permita desarrollar habilidades y competencias similares a las que encontrarán y requerirán en situaciones de la vida cotidiana o profesional; sin embargo, el Aprendizaje Basado en Problemas es una metodología activa de aprendizaje que promueve en estudiantes de educación superior la identificación y análisis de problemas, planteamiento de hipótesis de solución, el acopio de información, la valoración de las soluciones planteadas y la extrapolación a la vida diaria (Díaz Barriga, 2006).

\section{Inteligencia Emocional.}

A partir de la primera publicación de Gardner (1983) Frames of Mind, se reformuló el concepto de Inteligencia, donde se establece que los seres humanos poseen siete tipos de inteligencia, cada una independiente de las otras. (Trujillo \& Rivas, 2005) inició así todo un cambio en la perspectiva acerca de lo que se entiende por Inteligencia como tal. Salovey y Mayer (1990), citado en Gabel, (2005), estructuraron el concepto de Inteligencia Emocional a partir de los aportes de Gardner en relación a los conceptos de Inteligencia Intrapersonal e Interpersonal. Años más tarde, Daniel Goleman define la Inteligencia Emocional como "la capacidad para reconocer nuestros propios sentimientos y los de los demás, para motivarse y gestionar la emocionalidad en nosotros mismos y en las relaciones interpersonales" (García Fernández \& Gimenez-Maz, 2010, p. 45). Bar-On en 1997, se refirieron a la Inteligencia Emocional como un conjunto de habilidades personales, emocionales, sociales y de destrezas que influyen en nuestra habilidad para adaptarse y enfrentar a las demandas y presiones del medio (Ugarriza, 2001).

Actualmente, este concepto toma mayor preponderancia considerando el efecto de la globalización donde se busca que las personas desarrollen habilidades y competencias que les permiten un desenvolvimiento pertinente acorde a las demandas de un entorno dinámico y cambiante dando énfasis en el rol que cumplen las emociones en las acciones de los individuos.

En tal sentido, a partir de las definiciones presentadas podemos concluir que la Inteligencia Emocional es la capacidad que tiene el individuo para poder reconocer y hacer uso de sus propias emociones de modo que pueda lograr una interacción adecuada con su medio y esto a su vez le permita adaptarse y establecer relaciones de manera saludable. 


\section{Modelo de Bar-On sobre la Inteligencia emocional y sus dimensiones.}

El presente estudio tuvo por finalidad determinar el efecto del ABP sobre el desarrollo de la Inteligencia Emocional (IE), es decir, sobre la funcionalidad de los individuos y la forma en cómo interactúan entre sí. Para ello, tomó como base los estudios realizados por Bar-On.

Este modelo de inteligencias no cognitivas planteó que estas habilidades y destrezas se desarrollan y cambian a lo largo del tiempo y pueden mejorar con el entrenamiento de modo que ser emocional y socialmente inteligente significa reconocer y expresar las emociones, comprenderse a sí mismos, actualizar sus capacidades potenciales, llevar una vida regularmente saludable y feliz. Son capaces de comprender la manera como las otras personas se sienten, de tener y mantener relaciones interpersonales satisfactorias y responsables, sin llegar a ser dependientes de los demás (Bar-On, 1997). Dicho de otro modo, la inteligencia emocional se convirtió en un factor importante en la determinación de nuestra habilidad para tener éxito en la vida, ya que influye en nuestro bienestar emocional general (Ugarriza y Pajares, 2005) en ese sentido, las personas que son más saludables y tienen un adecuado funcionamiento y éxito en la vida son consideradas emocionalmente inteligentes.

Bar-On (1997), propuso cinco componentes que se detallan a continuación:

1. Componente intrapersonal (CIA): Evalúa la auto identificación general del individuo, la autoconciencia emocional, asertividad, autorrealización e independencia emocional.

2. Componente interpersonal (CIE): Evalúa la empatía, la responsabilidad social, las relaciones sociales.

3. Componente de adaptabilidad (CAD): Se refiere a la capacidad del individuo para evaluar correctamente la realidad y ajustarse de manera eficiente a nuevas situaciones, así como para crear soluciones adecuadas a los problemas diarios. Incluye las nociones de prueba de la realidad, flexibilidad y capacidad para solucionar problemas.

4. Componente de manejo de emociones (CME): Considera la capacidad para tolerar presiones y la capacidad de controlar impulsos.

5. Componente de estado de ánimo en general $(C A G)$ : Evalúa el optimismo y la felicidad.

\section{Importancia de la Inteligencia Emocional y el desarrollo de las Habilidades Blandas en el contexto de la Educación Superior.}

El desarrollo de competencias en los estudiantes, especialmente las competencias emocionales, se ha convertido en un tema de gran relevancia al momento de optar por mayores oportunidades laborales Singer, Guzmán y Donoso (2009).

Según Fiszbein, Cosentino y Cumsille (2016) "las habilidades socioemocionales, también denominadas habilidades blandas, son un amplio conjunto de habilidades y son relevantes para cualquier tipo de trabajo" (p.4). Singer, Guzmán y Donoso (2009) las denominaron como un "conjunto de habilidades no cognitivas esenciales para aprender y desempeñarse exitosamente en el trabajo" (p.2). En ese sentido, se destacó la contribución de la educación en el desarrollo de habilidades y capacidades del estudiante para su integración en la sociedad y su consecuente inserción laboral Matus y Gutiérrez (2015).

Vargas y Carzoglio (2017), recopilaron e identificaron las siguientes habilidades, asociadas a la Inteligencia Emocional, como las más demandadas por las empresas en la actualidad. 
- Capacidad para interactuar efectivamente, comunicar mensajes, ser persuasivo.

- Solución de problemas, que implica capacidad de percepción y análisis, así como delinear soluciones.

- Trabajar bajo parámetros, entender sus límites y las decisiones a tomar.

- Apertura al cambio y la actualización constante.

- Pensamiento innovador.

De esa forma, se observó la necesidad de una educación superior que busque preparar al desenvolvimiento de los estudiantes en el escenario laboral. Bajo esta misma línea, el Banco Interamericano de Desarrollo (BID) en el año 2017 hizo referencia a un estudio realizado durante ese año, se halló que diversos programas educativos latinoamericanos necesitan fortalecer aún más las habilidades socioemocionales que faciliten la inserción laboral.

El acceso a la educación en nuestra región ha ido en aumento en los últimos años, sin embargo, ello no significa que los jóvenes hayan desarrollado las competencias personales necesarias para hacer frente al mundo laboral Vargas y Carzoglio (2017).

Así mismo, a nivel nacional, Semana Económica (2014), citada en Aparicio y Medina, (2015) avaló una investigación sobre las habilidades consideradas como más importantes, encontrándose que el Liderazgo, Planificación Estratégica, Prospectiva, Toma de decisiones, Impacto e Influencia, Conocimiento del Negocio, Orientación al Logro, Empowerment, Desarrollo de personas y dirección de equipos de trabajo son las mejores ubicadas. En este sentido, el Ministerio de Educación (2015) tiene como objetivo fundamental que los estudiantes logren aprendizajes pertinentes y de calidad, y a su vez que ello sea favorable para el desarrollo y la competitividad nacional. Es decir, resaltan las acciones que fomenten e incentiven el desarrollo del aprendizaje cognitivo y también no cognitivo, lo cual engloba las habilidades blandas que se relacionan de manera muy directa con la inteligencia emocional.

En base a lo descrito, se considera esencial plantear y ejecutar procesos de enseñanzaaprendizaje que permitan fortalecer las competencias y/o habilidades que son las más requeridas en el contexto laboral de modo que como resultado tengamos estudiantes emocionalmente inteligentes $\mathrm{y}$, por ende, mucho más empleables. Considerando que tanto la inteligencia emocional como el uso de las habilidades sociales proveen a los alumnos de recursos necesarios para su desarrollo personal, se busca entonces generar un programa de entrenamiento en habilidades sociales e inteligencia emocional que unifique el uso de estos y los componentes principales en los que todos los autores mencionados coinciden.

\section{Método}

La presente investigación corresponderá al tipo aplicado, empleando el método experimental (McMillan \& Schumacher, 2010), dado que se manipulará la variable independiente (Aprendizaje Basado en Problemas) para que produzca un efecto sobre la variable dependiente (Inteligencia Emocional), la cuál será medida (Hernández, Fernández \& Baptista, 2010). El diseño es cuasiexperimental (Hernández, Fernández \& Baptista, 2010), se empleará grupos de sujetos intactos previamente establecidos, pasarán por un pretest, luego se les administrará la condición de tratamiento (presencia o ausencia) a cada grupo y finalmente pasarán por el postest (McMillan $\&$ Schumacher, 2010). 


\section{Participantes}

La población está conformada por los 1281 ingresantes a la universidad privada de Lima, en donde se realizó la investigación. La muestra consta de 48 ingresantes a la Carrera de Administración de Empresas del turno de la mañana, matriculados en el curso de Desarrollo de la Inteligencia Emocional I. De estos, 24 conformaron el grupo experimental y los otros 24 el grupo control. El muestreo fue no probabilístico e intencional, asegurándose la homogeneidad en ambos grupos en cuanto a proporciones de género en cada grupo y edades similares. Los criterios de inclusión contemplaron a los estudiantes mencionados, que poseían entre 16 y 21 años. Como criterios de exclusión se tomó en cuenta a los estudiantes mayores de 21 años y a los que no rindieron la evaluación de pretest y/o postest.

\section{Instrumento}

La variable Inteligencia Emocional se midió con el test del Inventario de Inteligencia Emocional de Bar-On (EQ-I Bar-On Emotional Quotient Inventory), el cual, desde una visión sistémica (personal, emocional y social) considera cinco componentes mayores de la inteligencia emocional: intrapersonal, interpersonal, adaptabilidad, manejo del estrés y estado de ánimo, que se relacionan lógica y estadísticamente, a través de puntuaciones dadas a cada uno de los 133 ítems que componen dicho test (formato de escala de respuesta tipo Likert, de autoclasificación de cinco puntos) (Ugarriza, 2001).

Para la validez del presente instrumento BarOn (1997) realizó un análisis factorial exploratorio de los ítems (método de extracción de componentes principales con rotación varimax) para lograr una mayor comprensión del concepto sobre el cual se basaba el inventario (inteligencia no cognitiva). Se llevaron a cabo diversos procedimientos de análisis de factores, tanto exploratorios como confirmatorios, para dar mayor solidez al proceso de desarrollo del inventario y para examinar la composición teórica de las diversas escalas y, asegurar, por lo tanto, la validez de constructo del presente test. A nivel nacional, Ugarriza (2001) realizó un análisis factorial confirmatorio de segundo orden y prueba Chi cuadrado para verificar la estructura propuesta por Bar-ON, comprobándose la misma estructura factorial. En cuanto a los estudios de confiabilidad ejecutados sobre el presente instrumento, se centraron en la consistencia interna y la confiabilidad retest. Bar-On (1997) reportó la consistencia interna a través del alfa de Cronbach entre .69 y .86 y confiabilidad retest de .85. A nivel nacional, los estudios de Ugarriza (2001) presentan una consistencia interna para el inventario total muy alto (coeficiente alfa de Cronbach de 0.93).

\section{Procedimiento}

A partir de lo propuesto por Diaz Barriga y Hernández (2010), para la implementación del ABP en aula, fue necesario cumplir con dos fases previas: se preparó la implementación del del ABP en aula, identificándose los propósitos a alcanzar con el uso de esta metodología, así como la elaboración del sílabo y los instrumentos de evaluación, acordes con las dimensiones de la Inteligencia Emocional que se buscaban potenciar. Asimismo, se preparó a los estudiantes expuestos a la intervención con el ABP, es decir, se realizó la presentación y explicación de los instrumentos de evaluación bajo esta metodología, así como la formación de equipos de trabajo con características similares: cada grupo estuvo conformado por 5 o 6 integrantes, con distribución de participantes tomándose en cuenta género y edad, y con la presencia de por lo menos un líder por grupo.

Se desarrolló la intervención en el grupo experimental a través de diseños de instrucción basados en el Aprendizaje Basado en Problemas, que pretende generar aprendizajes significativos 
para el desarrollo de las capacidades de la inteligencia emocional. Se desarrollaron 16 sesiones de aprendizaje, de 02 horas pedagógicas cada una, con intervención pedagógica grupal en el marco del curso titulado: "Curso de Desarrollo Personal I", que forma parte de la malla curricular de los estudiantes del primer semestre de la universidad donde se realizó la investigación. En el caso del grupo de control, también se realizaron las 16 sesiones de aprendizaje en el marco del mismo curso, pero se utilizaron metodologías de aprendizaje tradicionales. Se aplicó el test de Inventario de Inteligencia Emocional de BarOn como pretest y postest para ambos grupos.

\section{Resultados}

A partir de las respuestas de los participantes a los reactivos del test del Inventario de Inteligencia Emocional se calcularon los puntajes directos (suma ponderada de ítems) para cada componente o capacidad emocional (Intrapersonal, Interpersonal, Adaptabilidad, Manejo del estrés y Estado de Ánimo General) de la variable dependiente Inteligencia Emocional.

Cabe indicar, que considerando la prueba de normalidad de Shapiro Wilks (W) para muestras similares al presente estudio (Razali \&Wah, 2011), las distribuciones de los puntajes en todos los componentes de la variable de estudio fueron normales, por ende, se emplearon estadísticos paramétricos para el contraste de hipótesis.

Tabla 2.

Análisis inferencial de las diferencias en los componentes de la inteligencia emocional entre el grupo experimental y el grupo control en el Pre-test

\begin{tabular}{lcccccc}
\hline Variable & Grupo & $n$ & $M$ & $D E$ & $t$ & $p$ \\
\hline \multirow{2}{*}{ Intrapersonal } & Experimental & 22 & 148.57 & 20.27 & -0.429 & 0.670 \\
& Control & 22 & 145.91 & 21.27 & & \\
Interpersonal & Experimental & 22 & 110.39 & 12.98 & -1.039 & 0.305 \\
& Control & 22 & 105.73 & 16.95 & & \\
Adaptabilidad & Experimental & 22 & 87.48 & 9.87 & 0.543 & 0.590 \\
& Control & 22 & 89.09 & 10.06 & & \\
Manejo de Estrés & Experimental & 22 & 63.65 & 9.74 & 0.154 & 0.879 \\
& Control & 22 & 64.14 & 11.39 & & \\
Estado de ánimo & Experimental & 22 & 66.43 & 7.05 & -1.212 & 0.232 \\
en general & Control & 22 & 63.05 & 11.32 & & \\
\hline
\end{tabular}

Nota. $n$ : número. M: Media. Md: Mediana. DE: Desviación Estándar. $t$ : T de Student para muestras independientes.

En el Pre-test no se encontraron diferencias significativas entre las puntuaciones promedio obtenidas por el grupo experimental $(n=22)$ y el grupo de control $(n=22)$ en los cinco componentes de la inteligencia emocional. Los valores $\mathrm{T}$ de Student para muestras independientes calculados para los componentes de la inteligencia emocional (Interpersonal $t=-0.429$, Interpersonal $\mathrm{t}=-1.039$, Adaptabilidad $\mathrm{t}=0.543$, Manejo del estrés $\mathrm{t}=0.154$, Estado de ánimo general $t=-1.212)$ fueron no significativos $(p>0.05)$, por ende se establece que no existen diferencias estadísticamente significativas trabajando con un nivel de confianza del 95 por 100, entre las medias (M) del grupo experimental (GE) y del grupo de control (GC) (Shaughnessy, 
Zechmeister y Zechmeister, 2007). Los resultados obtenidos en el Pre-test garantizan la homogeneidad de las puntuaciones en las variables de estudio entre los grupos y esta condición básica permite asumir que los análisis posteriores se basarán en el hecho de que las diferentes condiciones experimentales parten de una puntuación equivalente en los componentes de la inteligencia emocional (Cubo, Martín y Ramos, 2011). Así los cambios que se generen posteriormente podrán ser atribuidos a la manipulación ejercida sobre la variable independiente (Programa basado en ABP) y no a una situación de partida diferente, en ambos grupos, en relación a su inteligencia emocional.

Tabla 3.

Análisis inferencial de las diferencias entre el Pre-test y el Post-test en los componentes de la inteligencia emocional en el grupo experimental y en el grupo de control.

\begin{tabular}{llllllllllll}
\hline & & \multicolumn{9}{c}{ Pre-Test } & \multicolumn{7}{c}{ Post-Test } & \multicolumn{7}{c}{ Diferencias emparejadas } \\
\hline Componente & Grupo & $\mathrm{n}$ & $\mathrm{M}$ & $\mathrm{DE}$ & $\mathrm{M}$ & $\mathrm{DE}$ & $\mathrm{M}$ & $\mathrm{DE}$ & $\mathrm{t}$ & $\mathrm{p}$ \\
Intrapersonal & GE & 22 & 148.57 & 20.27 & 158.04 & 13.41 & .52 & 16.81 & 2.09 & 0.04 \\
& GC & 22 & 145.91 & 21.27 & 147.36 & 20.41 & -1.45 & 18.53 & -0.37 & 0.72 \\
Interpersonal & GE & 22 & 110.39 & 12.98 & 117.17 & 9.03 & 3.22 & 10.54 & 2.11 & 0.03 \\
& GC & 22 & 105.73 & 16.95 & 108.14 & 16.16 & -2.41 & 11.50 & -0.98 & 0.34 \\
Adaptabilidad & GE & 22 & 87.48 & 9.87 & 89.43 & 8.28 & 2.04 & 8.04 & 2.10 & 0.03 \\
& GC & 22 & 89.09 & 10.06 & 89.05 & 11.57 & .05 & 10.77 & 0.02 & 0.98 \\
Manejo Estrés & GE & 22 & 63.65 & 9.74 & 70.83 & 9.55 & 2.83 & 7.20 & 2.09 & 0.04 \\
& GC & 22 & 64.14 & 11.39 & 63.23 & 11.27 & .91 & 9.05 & 0.47 & 0.64 \\
Estado ánimo 1 & GE & 22 & 66.43 & 7.05 & 67.48 & 6.27 & 1.04 & 4.72 & 2.10 & 0.03 \\
General & GC & 22 & 63.05 & 11.32 & 65.50 & 7.70 & -2.45 & 9.17 & -1.26 & 0.22 \\
& & & & & & & & & & &
\end{tabular}

Nota. n: número. M: Media. DE: Desviación Estándar. t: T de Student para muestras relacionadas.

El análisis Pretest-Postest en el grupo control $(\mathrm{GC})(\mathrm{n}=22)$ garantiza la equivalencia de las puntuaciones en las mediciones de la variable dependiente (inteligencia emocional) en ausencia de la variable independiente (Programa de ABP). Esta homogeneidad ofrece la seguridad en cuanto a la ausencia de variables extrañas que hayan contaminado el estudio y evidencia validez interna en cuanto al diseño de investigación (Cubo et al, 2011). Así se observa que no existen diferencias estadísticamente significativas, trabajando con un nivel de confianza del 95 por 100, en el grupo de control en las mediciones de los componentes de la inteligencia emocional entre el Pretest y el Postest y en ausencia del Programa de ABP.

El análisis del Pretest- Postest en el grupo experimental (GE) $(n=22)$ evidencia los cambios generados por la variable independiente. A partir de los resultados del grupo experimental en los componentes de la inteligencia emocional antes y después de la exposición al Programa en ABP llevan a determinar que existen diferencias significativas entre las medias de ambas mediciones por efecto de la variable independiente en el grupo experimental, trabajando con un nivel de confianza del 95 por 100 (Cubo et al., 2011). Al compararse las diferencias entre las medias de los grupos en el componente Intrapersonal, se encontraron diferencias estadísticamente significativas en el grupo experimental (Pre-Test $\mathrm{M}=148.57$; Post-Test $\mathrm{M}=$ 158.04; $\mathrm{t}=2.09 ; \mathrm{p}=.04)$, encontrándose mejoría. En cuanto al componente Interpersonal también se encontraron diferencias estadísticamente significativas en el grupo experimental (Pre-Test M 
$=110.39 ;$ Post-Test $\mathrm{M}=117.17 ; \mathrm{t}=2.11 ; \mathrm{p}=.03)$, encontrándose un incremento en este aspecto. Del mismo modo en el componente Adaptabilidad se observan diferencias (Pre-Test $\mathrm{M}=87.48$; Post-Test $\mathrm{M}=89.43 ; \mathrm{t}=2.10 ; \mathrm{p}=.03$ ), encontrándose un aumento. En el caso del componente Manejo del Estrés se encuentran diferencias estadísticamente significativas (Pre-Test $\mathrm{M}=63.65$; Post-Test $\mathrm{M}=70.83 ; \mathrm{t}=2.09 ; \mathrm{p}=.04)$, encontrándose mejoría. En el componente Estado de Ánimo General también se encontraron diferencias (Pre-Test $\mathrm{M}=66.43$; Post-Test $\mathrm{M}=67.48$; $\mathrm{t}$ $=2.10 ; \mathrm{p}=.03$ ). Considerando la tendencia de los resultados observados y presentados en la tabla 2, se infiere que el Programa de ABP es útil para desarrollar los componentes de la inteligencia emocional.

\section{Discusión}

El presente estudio tuvo como objetivo identificar, analizar y comparar el efecto del Aprendizaje Basado en Problemas (ABP) en la Inteligencia Emocional (IE) en estudiantes de primer semestre de una universidad privada, el cual fue alcanzado y se presentaron los resultados. En esta sección se analizaron y explicaron los resultados obtenidos en el marco de los antecedentes y las bases teóricas de las variables desde cada uno de sus componentes.

Con respecto al componente intrapersonal de la inteligencia emocional las diferencias intragrupo solo presentaron un cambio estadísticamente significativo en las puntuaciones en el grupo experimental. Por ende, considerando la comparación intergrupo (experimental y control) e intragrupo, en las fases de pretest y postest, se cumplen los supuestos y se evidencia el efecto de la intervención que emplea el $\mathrm{ABP}$ en sesiones de desarrollo del componente intrapersonal en estos participantes. Cabe resaltar que la capacidad intrapersonal, entendida como la conciencia de sí mismo, la asertividad, el autoconcepto y la autorrealización e independencia que posee un individuo pueden ser desarrolladas considerando las etapas de la aplicación del ABP. Tal como mencionó Pérez (2018) esta es una metodología que dota de autonomía al estudiante, pues desarrolla en él una actitud de compromiso con su propio aprendizaje, lo lleva a la reflexión constante y a pensar sobre el proceso por el cual construye sus capacidades, mediante actividades tales como la discusión, la argumentación, la evaluación de uno y de sus pares.

En referencia al componente interpersonal de la Inteligencia Emocional se evidenciaron diferencias en las fases del pretest y postest en el grupo experimental luego de la aplicación del $\mathrm{ABP}$, a comparación del grupo de control, en quienes no se encontraron cambios significativos. En el marco de las competencias o habilidades que propone la educación se busca desarrollar la capacidad empática y la cooperación del educando para que este, tienda a establecer relaciones interpersonales satisfactorias. Tal como plantean Delgado y de Justo (2018) el ABP exige que frente a un problema los estudiantes tengan que constituirse e integrarse a grupos para trabajar en equipo, participando de un proceso cooperativo y de interdependencia positiva, y anteponiendo sus necesidades y perspectivas a la de los otros, esto implica un diálogo constaste y creación de consensos en función a la tarea o meta en conjunto. En este sentido, el ABP combina la adquisición de conocimientos con el desarrollo de habilidades, actitudes y competencias interpersonales que son útiles en el mercado profesional (Roblego, Fidalgo, Arias \& Álvarez, 2015).

En cuanto al componente adaptabilidad de la Inteligencia Emocional (IE) se evidenciaron diferencias estadísticamente significativas en las fases del pretest y postest en el grupo experimental luego de la aplicación del ABP, a comparación del grupo de control, en el que no se encontraron cambios significativos. Esto podría explicarse a partir de los estudios de GregoriGiralt y Menéndez-Varela (2015), quienes encontraron que el ABP, como método, permite abordar problemas cotidianos y a su vez constituye una estrategia que promueve de forma 
individual y grupal el aprendizaje de habilidades para la superación de obstáculos en la vida real. Esta capacidad de afronte implica la identificación, selección e integración apropiada de conocimientos previos, recursos y habilidades, que conllevan a que el sujeto frente a un problema bajo el método $\mathrm{ABP}$ tenga que integrar sus aprendizajes en un todo coherente en función a la naturaleza del problema (García-Castro, Ruiz-Ortega \& Mazuera-Ayala, 2018).

A partir de los resultados presentados, se observó que el componente manejo del estrés de la Inteligencia Emocional, mostró en los análisis intragrupo e intergrupo, en las fases de pretest y postest solo cambios estadísticamente significativos en las puntuaciones en el grupo experimental. El poseer los rasgos para afrontar situaciones adversas y controlar las propias emociones, constituye capacidades que permiten la adaptación del sujeto a situaciones sociales y de aprendizaje. Es así, que en el presente estudio se evidencia que el ABP tiene un efecto positivo sobre dicho componente, ya que el empleo de esta metodología, tal como plantea Gregori-Giralt y Menéndez-Varela (2015), permite adecuar una problemática a la realidad de un estudiante ingresante, contextualizándolo en su naturaleza temática y poder asignarle un nivel de dificultad acorde al perfil del aprendiz. Sin embargo, la exposición al ABP conlleva al estudiante a afrontar el desafío de adaptarse a una nueva metodología, trabajar en equipo, gestionar información, asumir codependencia positiva en el proceso y el producto y ganar autonomía, se convierten en potenciales estresores, con los cuales el estudiante tendrá que lidiar para cumplir eficaz y eficientemente las actividades y tareas (Solaz-Portolés, San José \& Gómez, 2011).

Asimismo, los resultados evidenciaron diferencias estadísticamente significativas para el componente estado de ánimo general, en las fases del pretest y postest en el grupo experimental luego de la aplicación del ABP, a comparación del grupo de control, en el que no se encontraron cambios significativos. Lo hallado coincide con lo planteado por Morales (2009), quien explica que el desempeño y los logros en cada paso de aplicación del ABP, en los cuales se experimentan éxitos y/o fracasos, hacen que el estudiante vaya aprendiendo una dinámica de trabajo que le exige actividades colaborativas (no competitivas) y de adecuada interacción entre pares (no individualistas), que finalmente determinarán la atribución causal de los resultados obtenidos como éxito (meta lograda) o fracaso (meta no lograda), siendo aquellas las que llevan a experimentar emociones positivas, tales como la felicidad, la alegría y el optimismo frente a uno mismo, las propias capacidades y los otros. Así, las experiencias de éxito en la clase promueven el optimismo de éxitos futuros de aprendizaje, que involucren un trabajo con pares. Con respecto a este último, diversos estudios enfatizaron la importancia del desarrollo de la confianza y el optimismo en estudiantes universitarios sobre las expectativas de sus logros como competencias requeridas y valoradas por el mercado laboral; por ende, su promoción desde la formación constituyen un valor de cara a su empleabilidad (Maldonado \& Yusit, 2013; Peralta, Besio, Rubio, Atabales \& Salinas, 2010; Cuadra-Peralta, Fuentes-Soto, Madueño-Soza, Veloso-Besio \& Meneses, 2012).

Finalmente, a partir de los resultados se concluye que el Aprendizaje Basado en Problemas produce efecto significativo en la inteligencia emocional en estudiantes de primer ciclo de una universidad privada. En tal sentido, la aplicación de dicho método desarrolla los componentes de la inteligencia emocional mejor que el método tradicional de enseñanza que se pretende en los estudiantes. Este estudio evidencia el valor del ABP como método o estrategia en el campo pedagógico no solo para el desarrollo de capacidades curriculares en relación a la enseñanza de ciencias y letras (habilidades duras), sino también para potenciar las habilidades blandas o capacidades personales relacionadas a la empleabilidad y la convivencia. En este sentido, se ha demostrado que la aplicación del ABP en el desarrollo de la inteligencia emocional potencia de manera integral al educando, más allá de lograr favorecer exclusivamente su 
empleabilidad: la sociedad requiere, más que nunca, una educación que forme individuos capaces de establecer una convivencia positiva, con los que lo rodean a nivel personal y profesional.

\section{Referencias}

Aparicio, V., \& Medina C. (2015). Habilidades Gerenciales que demandas las empresas en el Perú. Un análisis comparativo (Tesis de maestría). Universidad del Pacífico, Lima.

Banco Interamericano de Desarrollo. (2017). Aprender mejor: Políticas públicas para el desarrollo de habilidades. Recuperado de: https://publications.iadb.org/bitstream/handle/11319/8495/Aprender_mejor_\%20Politic as_publicas_para_el_desarrollo_de_habilidades.PDF?sequence $=1 \&$ isAllowed $=\mathrm{y}$

Bar-On, R. (1997). Development of the Baron EQ-I: A measure of emotional and social intelligence. Presentado en 105th Annual Convention of the American Psychological Association, Chicago.

Cuadra-Peralta, A. A., Fuentes-Soto, L. K., Madueño-Soza, D., Veloso-Besio, C. B., \& Meneses, Y. B. (2012). Mejorando clima organizacional y de aula, satisfacción vital y laboral. Fractal: Revista de Psicología, 24(1), 3-26. Doi: https://doi.org/10.1590/S198402922012000100002

Cubo, S., Martín, B., \& Ramos, J.L. (2011). Métodos de investigación y análisis de datos en ciencias sociales y de la salud. Madrid: Ediciones Pirámides.

Díaz Barriga, F. (2006). El enfoque de competencias en la educación ¿una alternativa o un disfraz de cambio? Perfiles Educativos, 28(111), 7-36. Recuperado de: http://www.scielo.org.mx/scielo.php?pid=S0185$26982006000100002 \&$ script=sci_arttext\&tlng=pt

Diaz Barriga, F., \& Hernández, G. (2010). Estrategias docentes para un aprendizaje significativo: una interpretación constructivista. México: McGraw-Hill.

Eggen, P., \& Kauchak, D. (2015). Estrategias docentes. Enseñanza de contenidos curriculares y desarrollo de habilidades de pensamiento. México: Fondo de Cultura Económica.

Escribano, A. \& Del Valle, A. (2010). El Aprendizaje Basado en Problemas (ABP). Una propuesta metodológica en Educación Superior. Madrid: Narcea Ediciones.

Delgado, A., \& de Justo, E. (2018). Evaluación del diseño, proceso y resultados de una asignatura técnica con aprendizaje basado en problemas. Educación XX1, 21(2), 179203. Doi: https://doi.org/10.5944/educxx1.19415

Fiszbein, A., Cosentino, C., \& Cumsille, B. (2006). El desafío del desarrollo de habilidades en América Latina: Un diagnóstico de los problemas y soluciones de política pública. Washington, DC: Diálogo Interamericano y Mathematica Policy Research.

Gabel, R. (2005). Inteligencia Emocional: Perspectivas y aplicaciones ocupacionales. Lima: Universidad ESAN. Recuperado de http://repositorio.esan.edu.pe/handle/ESAN/517

García-Castro, G.; Ruiz-Ortega, F., \& Mazuera-Ayala, A. (2018). Desarrollo de la argumentación y su relación con el ABP en estudiantes de ciencias de la salud. Revista Latinoamericana de Estudios Educativos, 14 (1), 82-94. Recuperado de: https://www.redalyc.org/articulo.oa?id=134156702005

García Fernandez, M., \& Giménez -Maz, S.I. (2010). La inteligencia emocional y sus principales modelos: propuesta de un modelo integrador. Espiral. Cuadernos del Profesorado, 3(6) 43-52. Doi: http://dx.doi.org/10.25115/ecp.v3i6.909

Gregori-Giralt, E., \& Menéndez-Varela, J. L. (2015). La evaluación en el Aprendizaje Basado en Problemas (ABP): Resultados de una experiencia didáctica en los estudios universitarios de Bellas Artes. Estudios pedagógicos (Valdivia), 41(2), 87-105. Doi: http://dx.doi.org/10.4067/S0718-07052015000200006

Gutiérrez, M., \& Expósito J. (2015). Autoconcepto, dificultades interpersonales, habilidades sociales y conductas asertivas en adolescentes. Revista Española de Orientación y $\begin{array}{llllll}\text { Psicopedagogía, } & 2(26), & 42 & - & 58 . & \text { Doi: }\end{array}$ https://doi.org/10.5944/reop.vol.26.num.2.2015.15215

Hernández, R., Fernández, C., \& Baptista, P. (2010). Metodología de la investigación (5ª ed.). México D.F.: McGraw Hill. 
Lino, R. (2015). Afronta-t: una experiencia innovadora con el alumnado de la facultad de psicología de la universidad de Málaga. Málaga: Universidad de Málaga.

Maldonado, G., \& Yusit, H. (2013). La satisfacción laboral como dimensión de la felicidad. Ciencia \& trabajo, 15(47), 94-102. Doi: http://dx.doi.org/10.4067/S071824492013000200010

Marra, R., Jonassen, D. H., Palmer, B. \& Luft, S. (2014). Why problem- based learning works: Theoretical foundations. Journal on Excellence in College Teaching, 25(3-4), 221-238. Recuperado de: https://www.albany.edu/cee/assets/Why_Problembased_learning_works.pdf

Matus, O., \& Gutierrez, A. (2015). Habilidades Blandas: Una ventaja competitiva en la formación tecnológica. GINT Journal of Industrial Neo-Technologies, 32-40. Recuperado de https://www.jint.usach.cl/sites/jint/files/art._9_print_v2n1jint006-15_v3.0_0.pdf

McMillan, J. H., \& Schumacher, S. (2005). Investigación educativa (5 $5^{\mathrm{a}}$ ed.). Madrid: Pearson Educación S.A.

Ministerio de Educación del Perú. (2015). Reglamento de Organización y Funciones. Recuperado de http://www.minedu.gob.pe/p/xtras/reglamento_de_organizacion_y_funciones_rof.pdf

Morales, P. (2009). Logros en motivación y el tercer nivel de estructura del conocimiento: un estudio empírico en contextos de aprendizaje correspondientes a una modalidad híbrida ABP. Revista Educación, 18 (34), 73-92. Recuperado: http://revistas.pucp.edu.pe/index.php/educacion/article/view/1681

Peralta, A. C., Besio, C. V., Rubio, Y. M., Atabales, L. R., \& Salinas, J. V. (2010). Efecto de un programa de psicología positiva e inteligencia emocional sobre la satisfacción laboral y vital. Salud \& Sociedad, I(2), 101-112. Doi: https://doi.org/10.22199/S07187475.2010.0002.00004

Pérez, L. (2018). El aprendizaje basado en problemas como estrategia didáctica en educación superior. Voces De La Educación, 3(6), 155-167. Recuperado: https://www.revista.vocesdelaeducacion.com.mx/index.php/voces/article/view/127

Razali, N., \& Wah, Y. (2011). Power Comparison of Shapiro-Wilk, Kolmogorov-Smirnov, Lilliefors and Anderson Darling tests. Journal of Statistical Modeling and Analytics, 2(1), 21-33. Recuperado de: http://www.de.ufpb.br/ ulisses/disciplinas/normality_tests_comparison.pdf

Robledo, P., Fidalgmo, R., Arias, O, \& Álvarez, M. (2015). Percepción de los estudiantes sobre el desarrollo de competencias a través de diferentes metodologías activas. Revista de Investigación Educativa, 33(2), 369-383. Doi: https://doi.org/10.6018/rie.33.2.201381

Shaughnessy, J.J., Zechmeister, E.B., \& Zechmeister, J.S. (2007). Métodos de investigación en psicología ( $7^{\mathrm{a}}$ ed.). México D.F.: McFraw-Hill Interamericana.

Singer, M., Guzmán, R., \& Donoso, P. (2009). Entrenando Competencias Blandas en Jóvenes (Proyecto $\left.\mathrm{N}^{\circ} 1080292\right)$. Chile: Pontificia Universidad Católica de Chile e INACAP. Recuperado de http://www.inacap.cl/tportal/portales/tp90b5f9d07o144/uploadImg/File/PDF/Entrenand o_Competencias_Blandas_en_Jovenes.pdf

Solaz-Portolés, J. J., San José, V., \& Gómez, Á. (2011). Aprendizaje basado en problemas en la Educación Superior: una metodología necesaria en la formación del profesorado. Didáctica de las ciencias experimentales y sociales. Didáctica de las Ciencias

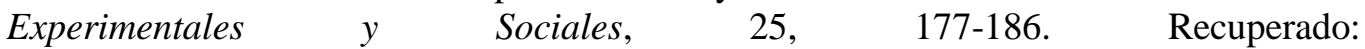
http://roderic.uv.es/handle/10550/21337

Ugarriza, N. (2001). La evaluación de la inteligencia emocional a través del inventario de BarOn (I-CE) en una muestra de Lima Metropolitana. Persona, 4, 129-160. Doi: https://doi.org/10.26439/persona2001.n004.817

Ugarriza, N., \& Pajares, L. (2005). La evaluación de la inteligencia emocional a través del inventario de BarOn ICE: NA, en una muestra de niños y adolescentes. Persona, 8, 1158. Doi: https://doi.org/10.26439/persona2005.n008.893

Vargas, F., \& Carzoglio, L. (2017). La brecha de habilidades para el trabajo en América Latina: Revisión y análisis en la región. Montevideo: OIT/Cinterfor. 